\title{
Table of cases
}

Abrahams $v$ Herbert Reiach Ltd

[1921] 1 KB 477 (CA) 70

Allen $v$ Toronto Star Newspapers Ltd

(1997) 142 DLR (4th) $518 \quad 37$,

\section{4}

Apple Corps Ltd and Apple

Computers Inc [1991] 3 CMLR

$49 \mathbf{1 9 2}$

Association des Journalistes

Indépendants du Québec (AJIQ) c

Cedrom-SNI [1999] RJQ 2753,

JQ no 4609 (QSC) $\mathbf{1 6 2}$

Bank of Credit and Commerce

International SA $v$ Ali and Others [2001] UKHL 8275

Bank of Credit and Commerce

International SA v Ali [2003] 3

All ER $51 \quad 275$

Banque Financière v Parc [1999] 1

AC $221 \quad 190$

Barclays Bank v Estates \&

Commercial Ltd [1997] 1 WLR

$425 \quad 78$

Barker v Stickney [1919] 1 KB 121

78

Bartsch v Metro-Goldwyn-Mayer Inc

391 F2d 150, 154-55 (2d Cir) cert denied 393 US 826, 89 S Ct 86

(1968) 149, 173, 174

$B G H$ vom 10 . Oktober 2002 - I ZR

180/00; BGH vom 19. Mai 2005

- I ZR 285/02 166, 173

Bourne Co $v$ Walt Disney Co $68 \mathrm{~F}$

3d 21 (2d Cir 1995 cert denied

116 SCt 1890, 1996) $\mathbf{1 3 7 , 1 7 3}$
Bowden Bros v Amalgamated

Pictorials Ltd [1911] 1 Ch 386

67

$B R T$ v SABAM [1974] ECR $51 \quad 192$

CCH Canadian Ltd v Law Society of

Upper Canada [2004] 1 SCR

339, 2004 SCC $13 \quad \mathbf{2 , 1 7 , 1 3 4 ,}$

\section{4}

Canadian Performing Right Society

Ltd v Famous Players Canadian

Corp (1927) 60 OR 280 (Ont SC)

$287 \quad 123$

Central Station Tribunal de première instance de Bruxelles (Brussels

Court of First Instance), 16

October 1996, Auteurs \& Media

1996, 426; Cour d'appel de

Bruxelles (Brussels Court of

Appeals), 28 October 1997,

Auteurs \& Media $1997 \quad 263$

Chavanel c Plurimedia et France 3

Tgi Strasbourg (2 ch com), 16 novembre $2001 \mathrm{SNJ} \mathbf{1 6 5}$

Chappell \& Co Ltd v Redwood Music Ltd [1981] RPC $337 \quad 77$

Clifford Davis $v$ WEA Records

[1975] 1 All ER 237 (CA) 131, 189

Colbern v Duncombe (1838) $9 \mathrm{Sim}$ $151 \quad 56$

Consorts Huston et autres $v$ Ste

Turner Entertainment (1991) 149

RIDA 197 (CA Versailles) $\mathbf{2 4 5}$

Copiepresse v Google case: Décision

du Tribunal de première instance 
de Bruxelles du 13 février 2007, n 06/10.928/C 169

Cox $v$ Land and Water Journal

Company (1869) LR 9 Eq 324

14, 61

Dansk Rekylriffel Syndikat

Aktieselskab v Snell [1908] $2 \mathrm{Ch}$ $127 \quad \mathbf{7 8}$

Dargaud $v$ Uderzo and SNE (11

January 2000) Case No Y 98-20$446 \mathbf{1 3 2}$

Denison v Ashdown (1897) 13 TLR $226 \quad 73$

Designer's Guild Ltd v Russell

Williams Textiles Ltd [2001] FSR

$11 \quad 213$

De Volkskrant No D 3.1294 (24

September 1997)(D Ct of

Amsterdam) tr (1998) 22

Columbia-VLAJLA 181 165,

166, 170, 171, 199, 203, 212

Director General of Fair Trading v

First National Bank plc [2000] 1

WLR 98, 109, on appeal [2000]

QB 672, on appeal to House of

Lords [2001] UKHL $52 \mathbf{1 3 0}$

Donaldson v Becket (1774) 2 Bro

PC 129 43-44, 52

Days Medical Aids Ltd v Pihsiang

Machinery Manufacturing Co Ltd

[2004] EWHC 44 (QB Comm Ct)

182, 185

Eastham v Newcastle United

Football Club Ltd [1964] $1 \mathrm{Ch}$

$413 \mathbf{1 8 4}$

Electronic Rights Defence

Committee (ERDC) c Southam

Inc 2009 QCCS 1473 [1999] JQ

no 349 (QBC) 162

Elton John \& Others $v$ Richard Leon

James \& Others [1991] FSR 397

183
Effect Associates $v$ Cohen 908 F2d 555 (USCA 9th Cir) (1990) 115 Emsland-Starke v Hauptzollampt Hamburg-Jonas [2002] ECR 111569192

Erskine MacDonald v Eyles [1921] 1

Ch 631 73-74

Euro-Excellence Inc v Kraft Canada

Inc 3 SCR 20, 2007 SCC $37 \quad 37$

Falcke v Gray (1859) 4 Drew 651

74

Farmer v Grant Richards (1904)

Mac CC 78; The Times (27 Feb

1904) $\mathbf{7 6}$

Freelens (5 July 2001) No I ZR

311/98 Federal Supreme Court

(Bundesgerichtshof) trans (2003)

34 IIC 227; (1997) No 308 O

284/96 (Regional Ct of Hamburg)

trans (1998) 22 Columbia-

VLAJLA 178 125, 166-68, 170, 199, 275

Fry v Lane (1888) 40 Ch D 312

188

General Association of Professional

Journalists v Central Station

[1988] ECC 40 (High Ct of

Brussels) 169

Gilliam v American Broadcasting Co (1976) 538 F 2d 14 (CA 2nd Cir) 150

Gledhow Autoparts $v$ Delaney

[1965] 1 WLR 1366182

Greenberg v National Geographic

Society 488 F3d 1331 (2007) and

497 F3d 1213 (2007) overruling

and vacating 244 F3d 1267 (11th

Cir 2001); en banc 2008 No. 05-

169642008 WL 2571333 (11th

Cir 2008) 148, 276

Griffith $v$ Tower Publishing Ltd

[1897] 1 Ch 2179 
Griggs Group Ltd v Evans (No 1) [2005] EWCA Civ $11 \quad \mathbf{1 3 5 , 1 3 9}$ Hall-Brown v Iliffe \& Sons Ltd (1910-1935) Mac CC 88 (20 Dec 1929) (Ch D) 63, 159

Hanseatische Oberlandesgericht Hamburg vom 24. Februar 2005 5 U 62/04 166, 173

Haywood v Brunswick Building

Society (1881) 8 QBD $403 \mathbf{7 8}$ Hogg v Kirby (1803) 43 Geo III; 8

Ves 215, $226 \quad 80$

Hole v Bradbury (1879) LR 12886

(ChD)(Fry J) 57, 66, 67

Hospital for Sick Children (Board of

Governors) v Walt Disney

Productions Inc [1968] Ch 52

9, 137, 194, 195, 198, 199, 277

In Re Waterson, Berlin and Snyder

Co 48 F 2dn 704 (1931) 78

In re Literary Works in Electronic

Databases Copyright Litigation

MDL 1379; F 3d 2007 WL

4197413 (2d Ct 2007) 153

Re Royal Institute of British

Architects (RIBA) Case ref

GP/908/March $2003 \quad 281$

Interfoto Picture Library Ltd $v$

Stiletto Visual Programmes Ltd

[1989] QB 433191

JC Williamson Ltd v MGM Theatres

Ltd (1937) 56 CLR 567 (Aust

HC) 194

Jeffreys $v$ Boosey (1854) 4 HLC 815

73

Johnson v Newnes [1894] 3 Ch 663

60

Ladbroke (Football) Ltd v William

Hill(Football) Ltd [1964] 1 All

ER 465 (HL) 17

LB (Plastics) Limited $v$ Swish

Products Limited (1979) RPC 551

(HL) 16
LC Page \& Co Inc v Fox Film Corp 83 F2d 196 (2d Cir 1936) 193

Leeds Rugby Ltd v Harris [2005] EWHC $1591 \mathbf{1 8 4}$

Lionel Sawkins v Hyperion Records [2004] EWHC 1530 (ChD)

(Patten J), aff'd [2005] 3 All ER 636 (CA) 30

Lucas $v$ Moncrieff (1905) Times LR 683 (Ch D) 56, 78-79, 291

Lloyds Bank Ltd v Bundy [1975] QB $236 \mathbf{1 8 9}$

Marx v Globe Newspaper Co 2002

(WL 31662569) 15 MLR 400

(Mass Sup 2002) 27

Mayhew v Maxwell (1860) 1 John \& H $31257,58,60,61,63,71$

Mazer v Stein 347 US 201 (1954) 219219

MCA Inc v Wilson 677 F 2d 180 (2d Cir 1981) 213

Morrissey v Procter \& Gamble Co 379 F 2d 375 (1967) $\mathbf{2 1 0}$ Millar v Taylor (1769) 98 Eng Rep 201 (KB) 52, 113, 205

National Westminster $v$ Morgan [1985] AC $686 \mathbf{1 8 8}$

Neufeld $v$ Chapman Hall (1901) The Times (31 Oct 1901) $\mathbf{7 5 , 7 6}$

Nichols $v$ The Amalgamated Press (1908) Mac CC $166 \quad 76$

Nordenfelt $v$ Maxim Nordenfelt [1894] AC $535 \mathbf{1 8 2}$

Osbourne v Dent [1925] 1 Ch 369 75

O'Sullivan v Management Agency and Music Ltd [1985] 3 All ER 351 159, 183

Panayiotou $v$ Sony Music

Entertainment (UK) Ltd [1994]

EMLR 229 183, 184

Pao On v Lau Yiu Long [1980] AC 614189 
Petty v Taylor (1897) 1 Ch $465 \quad 63$ Pettkus v Becker (1980) 2 SCR 834 190

Performing Right Society $v$ London

Theatre of Varieties [1924] AC 1

(HL) 74, 115, 124

Planché v Colburn (1831) 8

Bingham's Common Pleas 13

64

Plurimédia (3 February

1998)(Tribunal de Grande

Instance de Strasbourg -

Ordonnance de Référé

Commercial) tr (1998) 22

Columbia-VLAJLA 199 165,

171, 199

PRS v London Theatre of Varieties

[1924] AC $1 \quad \mathbf{1 1 5}$

Reade v Bentley (1857) $3 \mathrm{~K} \& \mathrm{~J} 271$;

(1858) 4 K \& J $656 \quad \mathbf{5 5 , 6 8 , ~} 275$

Re Drake Insurance [2001] Lloyd's

Rep IR $643 \mathbf{1 8 1}$

Reed Elsevier and others $v$

Muchnick and others (US Ct of

Appeals 2nd Ct, docket 08-203)

153

Re Grant Richards [1907] 2 KB 33

77-79

Re Judes' Musical Compilations

[1906] 2 Ch 595 67, 71

Re Royal Institute of British

Architects (RIBA) Case ref

GP/908/March $2003 \quad 281$

Re Writer's Union of Canada

Certification Application (1998)

84 CPR (3d) $329 \quad 119$

Rillon c Sté Capital Méda Cour de

cassation (1 chambre civile) 12

juin $2001 \mathbf{1 6 5}$

Robertson $v$ Thomson Corp (2001)

15 CPR 4th 147 (2001) 109

ACWS 3rd 137 (SCJ); (2004) 72

OR 3rd 481, 243 DLR 4th 257
(CA); 2006 SCC 43 [2006] 2

SCR 363, 274 DLR 4th 138

(SCC) 28, 75, 97, 134, 153,

160

Robertson v The Gale Group Inc

(Statement of Claim, Ont SCJ,

Toronto 24 July 2003) $\mathbf{1 6 1}$

Robertson v Proquest Information

Limited and Learning Company

and Others, Ct File No. 03-CV-

252945CPOB/OC/OD/A2 161

Robin Ray v Classic FM plc [1998]

ECC 488 (Ch D) 14, 196

Royal Bank of Scotland plc v Etridge

(No 2) [2001] UKHL $44 \mathbf{1 8 8}$

Ryan v Carl Corp 23 F 2d 1146,

1151 (ND Cal 1998) $\mathbf{1 4 7}$

Saphena Computing Ltd $v$ Allied

Collection Agencies Ltd

(1988)[1995] FSR 616 (QB)

appeal dismissed (1989)[1985]

FSR 649 (CA) 195-99

Schroeder v Macaulay [1974] 1

WLR 308 (CA) 159, 183, 188

Serra $v$ Famous-Lasky Service Ltd

(1922) 127 LT 109 (CA) 193

Sims v Marryat (1851) 17 QB 279

\section{3-74}

Slumber-Magic Adjustable Bed Co v

Sleep-King Adjustable Bed Co

(1984) 3 CPR (3d) 81 (BCSC)

17

Smith v Johnson (1863) 4 Giff 632

57

Stevens $v$ Benning (1855) $1 \mathrm{~K} \& \mathrm{~J}$

$168 \mathbf{6 6}$

Sweet v Cater (1841) 11 Sim 572

68

Tam Wing Chuen v Bank of Credit and Commerce Hong Kong Ltd [1996] 2 Butterworths Co L

Cases $69 \quad \mathbf{1 8 1}$ 
Tasini v New York Times Co 972 F

Supp 804 (NYSD Ct 1997); 184 F 2d 350 (NYSD Ct 2001); $981 \mathrm{~F}$ Supp 841 (NYSD Ct 2001); 206 F3d 161 (US Ct of Apps (2nd Cir), 1999); 533 US 483, $121 \mathrm{~S}$ Ct 2381 (2001) 7, 10, 114, 115, 116, 141-62, 168, 170, 171, 176, 178, 203, 220, 221, 222, 228, $258,260,261,263,264,275$, 277-80, 282

Tasini $v$ New York Times 184 F 2d

350 (NY D Ct 2002) 352

\section{1-53}

Trade Auxiliary v Middlesbrough (1889) 40 Ch D $425 \quad 57$

Twentieth Century Music Corp v Aiken 422 US 151, 95 S Ct 2040 (1975) 147

Tymshare v Covell 727 F2d 1145 (DC Cir) (1984) 131

Union Eagle Ltd v Golden Achievement Ltd [1997] AC 514 191

Union of French Journalists and

National Syndicate of Journalists v SDV Plurimédia (FR) (3

February 1998) (Tribunal de

Grande Instance de Strasbourg Ordonnance de Référé

Commercial) tr (1998) 22

Columbia-VLAJLA $199 \mathbf{1 6 5}$

United States v Dubilier Condenser

Corporation 289 US 178 amended 289 US 706 (1933) 34

University of London Press Ltdv

University Tutorial Press Ltd

[1916] 2 Ch $601 \quad 17$

Urteil des Bundesgerichtshofs

(BGH) vom 19. Mai 2005, Az. I

ZR 285/02 173
Vedatech $v$ Crystal Decisions (UK) Ltd [2002] EWHC 818 (Ch D) 190

Walsh v Lonsdale (1881) $21 \mathrm{ChD} 9$

75

Walford v Miles [1992] 2 AC 128

130

Walter v Howe (1881) $17 \mathrm{ChD} 708$ 63

Ward Lock \& Co v Long (1918) 34 Times LR $351 \quad \mathbf{7 4}$

Wiener Gruppe Austrian Supreme

Court (osterreichisch Oberste Gerichtshof) 12 August 1998, Multimedia und Recht 1999

172

William v Lacey [1957] 1 WLR 932 190

Writers Guild of Canada v Canadian

Broadcasting Corporation [2006]

OJ No $2979 \mathbf{1 2 0}$

Wyatt v Barnard (1814) 3 V \& B 77

58

WWW v World Wrestling Foundation

[2002] EWCA 196 185-6

Yacht-Archiv Hanseatisches

Oberlandesgericht Hamburg vom

24. February 2005 - 5 U 62/04

173

Zang Tumb Tuum v Holly Johnson [1993] EMLR $61 \mathbf{1 8 3}$

Zweites Gesetz zur Regelung des

Urheberrechts in der

Informationsgesellschaft vom 26.

Oktober 2007, Bundesgesetzblatt

Jahrgang 2007 Teil I Nr. 54, 31.

Oktober 2007 123, 173

Zyla $v$ Wadsworth $360 \mathrm{~F} 3 \mathrm{~d}$

243(Mass 1st Cir)(2004) 151 